\title{
INNOVACIÓN METODOLÓGICA APLICADA A LA ENSEÑANZA DE SISTEMAS ELÉCTRICOS DE POTENCIA
}

\author{
Manuel Villarroel M. ${ }^{1} \quad$ César San Martín S. $^{1} \quad$ Jorge Chávez M. $^{1} \quad$ Ildefonso Harnisch V. ${ }^{2}$ \\ Recibido el 11 de marzo de 2004, aceptado el 10 de mayo de 2004
}

\begin{abstract}
RESUMEN
En este trabajo se presenta la implementación de un sitio WEB de apoyo a la docencia en la asignatura de Sistemas Eléctricos de Potencia (SEP) para las carreras de Ingeniería Eléctrica de la Universidad de La Frontera. Este sitio, si bien incluye material de apoyo a la asignatura como apuntes, ejercicios y actividades de laboratorio; contiene además un software de simulación numérica que permite resolver el problema de cálculo de flujos de potencia en SEP utilizando el método de Newton-Raphson. Las características de ser multiplataforma, robusto, flexible, con interfaz gráfica al usuario amigable y de accesibilidad vía Internet lo convierten en un material indispensable para los estudiantes. Esto se complementa con actividades de laboratorio en las cuales los alumnos experimentan con instrumentación asociada y las clases expositivas donde se presentan todos los conceptos asociados a los SEP. Esta innovación en la metodología de enseñanza en SEP mejora substantivamente el logro de los objetivos conceptuales perseguidos en la asignatura.
\end{abstract}

Palabras claves: Análisis de sistemas de potencia, flujo de carga, programas computacionales, enseñanza.

\begin{abstract}
This paper presents an implementation of a WEB site to support the course Electric Power Systems, which is taught in the Electrical Engineering Department at Universidad de la Frontera. This site includes support materials such as notes, exercises and laboratory activities, as well as a numerical simulation software that solves load flow problems in Electric Power Systems, using the Newton Raphson method. The site has a multi-platform style, robustness, flexibility and a user friendly graphic interface, which transforms it in an indispensable educational tool for electrical engineering students. All of the above is complemented with activities in the laboratory, where the main concepts associated to electric power systems are demonstrated with appropriate instrumentation, together with release of useful information. This innovation in the teaching of Electric Power Systems improves substantially the understanding of concepts associated with the course.
\end{abstract}

Keywords: Power system analysis, load flow, computer programs, education.

\section{INTRODUCCIÓN}

En el área de Ingeniería Eléctrica, uno de los campos más relevantes lo constituye el análisis de los Sistemas Eléctricos de Potencia (SEP). Los principales tópicos que se desarrollan son: modelación de la máquina sincrónica, regulación de tensión, cálculo de flujos de potencia, operación económica, estabilidad, entre otros. Sin duda, que no es posible entregar estos conceptos sólo de manera teórica y expositiva en el aula sin un apoyo de actividades de laboratorio y simulación. En sesiones de laboratorio, los estudiantes realizan actividades tendientes a consolidar conocimientos y experimentar con el equipamiento asociado. El principal problema que se tiene para ello, es la restricción impuesta al acceso de los estudiantes a los laboratorios debido a que no se cuenta con toda la infraestructura necesaria. El aumento de la infraestructura significaría un alto costo que las instituciones no están en condiciones de absorber. Por otra parte, no es fácil realizar alteraciones por parte de los estudiantes en las configuraciones, en valores de carga, en variaciones de tensión, etc., ya que la manipulación sin una adecuada supervisión representa un alto riesgo sobre el equipamiento y las personas, debido a los elevados valores de voltaje, corriente y potencia, característicos de los SEP. Por esto, resulta muy atractiva la simulación de SEP de modo de experimentar situaciones que en la práctica no es posible conseguir. Más aún, la versatilidad que presenta un software de acceder a él sin

\footnotetext{
${ }^{1}$ Universidad de La Frontera, Departamento de Ingeniería Eléctrica, manuelvi@ufro.cl

${ }^{2}$ Universidad de Tarapacá, Departamento de Electrónica, harnisch@uta.cl
} 
restricciones de tiempo, con una adecuada infraestructura de computadores en la institución, es sin lugar a dudas una gran ventaja para los estudiantes. Por otro lado, este software que tiene las características de ser flexible, con una interfaz gráfica al usuario (GUI) amigable, multiplataforma, robusto, accesible desde Internet y de licencia gratuita para la educación, se convierte en una herramienta poderosa con aportes muy significativos para la enseñanza de la Ingeniería Eléctrica.

En este trabajo se presenta un software de simulación para el cálculo de flujos de potencia en SEP desarrollado en JAVA [7] que, junto al diseño de un sitio WEB que contiene material de apoyo como apuntes, ejercicios y prácticas de laboratorio, innovan significativamente la enseñanza en SEP para los estudiantes de las carreras de Ingeniería Eléctrica de la Universidad de La Frontera.

\section{DESCRIPCIÓN DEL SITIO WEB}

El diseño del sitio WEB está basado en una unidad central (página principal) [3], [6], [8], que contiene enlaces a varios tópicos principales, los que a su vez están divididos en unidades interrelacionadas entre ellas y la unidad central. La página principal, www.inele.ufro.cl/sep, se muestra en la Fig. 1 y posee todo lo necesario para abordar los temas de interés en SEP, contando para ello con dos frames. El primero incluye vínculos a información detallada del WEB de SEP, como programas de simulación, apuntes del curso, ejercicios propuestos, espacio para envío de dudas y consultas, dando con esto un entorno con variadas opciones que el usuario puede explorar. Cada una de estas páginas cuenta con una organización similar a la principal, con la salvedad de que las que derivan de ellas están interrelacionadas entre sí y con su primaria correspondiente, de esta forma se asegura cierta jerarquía en el sitio. El segundo frame da a conocer la descripción de la asignatura, los objetivos generales, recursos metodológicos y los contenidos del curso parte teórica y práctica experimental. La jerarquía de contenidos en el sitio WEB se muestra en la Fig. 3 donde se observan las posibles rutas de navegación en el sitio. Las páginas que continúan a la principal son: Apuntes, que contiene archivos PDF; C.F.P que contiene el simulador de cálculo de flujo de potencia (Fig. 2); Ejercicios resueltos y propuestos en formato PDF; y Dudas y/o Comentarios que corresponde a la base de datos de preguntas más frecuentes (FAQ).

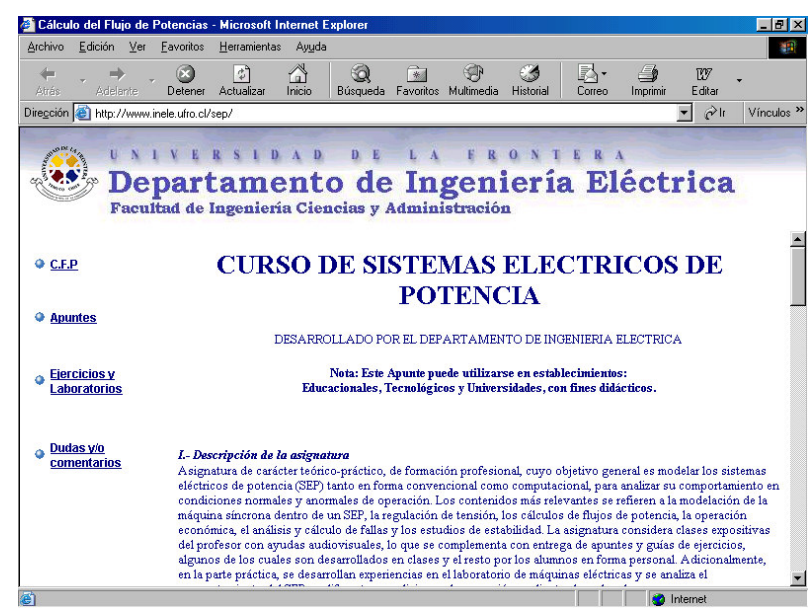

Fig. 1.- Algunas páginas del sitio WEB: página principal

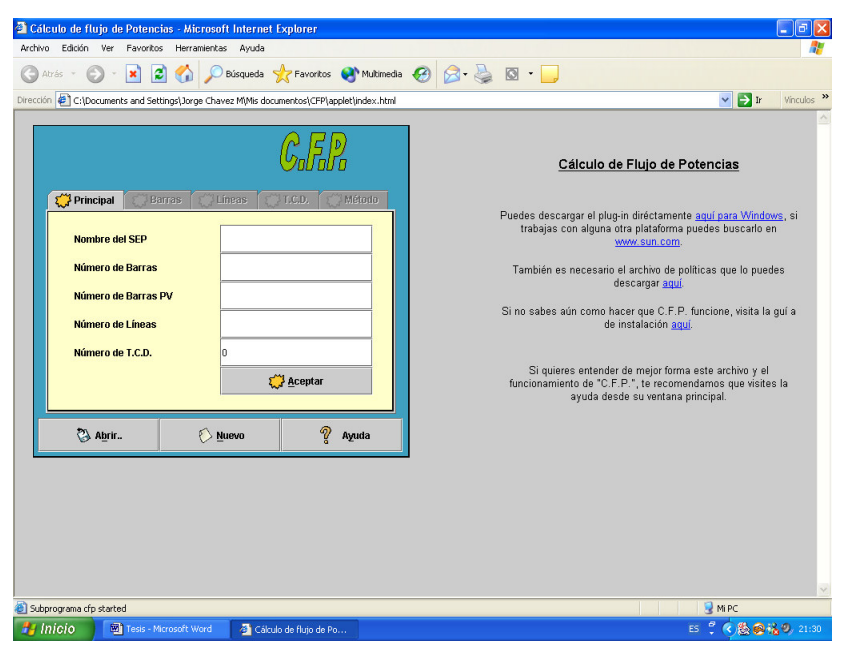

Fig. 2.- Algunas páginas del sitio WEB: simulador C.F.P 


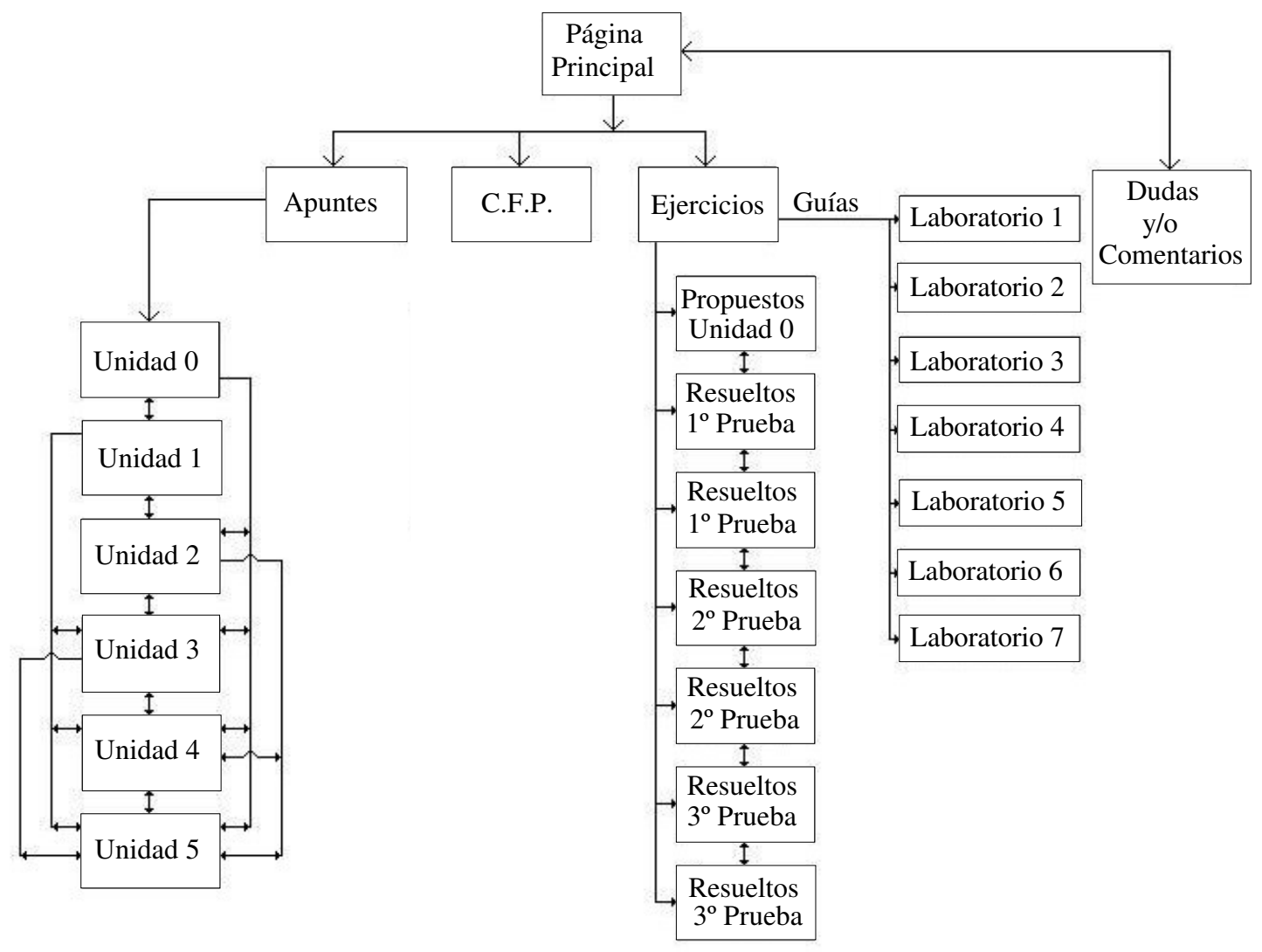

Fig. 3.- Distribución de la información en el servidor WEB

\section{EL SOFTWARE DE CÁLCULO DE FLUJOS DE POTENCIA}

El Análisis del Flujo de Potencias (AFP) [1], [2], [4] permite programar las ampliaciones necesarias del SEP y determinar su mejor modo de operación, teniendo en cuenta posibles nuevos consumos, nuevas líneas o nuevas centrales generadoras. Permite además estudiar los efectos sobre la distribución de potencias, cuando se producen pérdidas temporales de generación o circuitos de transmisión, como también ayudar a determinar los programas de despacho de carga para obtener un funcionamiento óptimo del sistema. Para el cálculo de flujos de potencia de sistemas eléctricos, se desarrolló el software C.F.P en JAVA [7] que utiliza el método de Newton Raphson en sus versiones: Jacobiano completo o Normal (Fig. 4), Desacoplado, Desacoplado Rápido y Jacobiano completo pero calculando la matriz Jacobiana cada cierto número de iteraciones [4], [5].

C.F.P es un software amigable que cuenta con ventanas de ayudas, salidas gráficas, validación de datos (alertando errores), tablas de resultados y guía de instalación. El programa entrega adicionalmente información que permite comparar los diferentes métodos en cuanto a las iteraciones realizadas y el listado de errores en función del número de iteraciones. Para ejecutar el programa se debe acceder desde la página principal de SEP, marcar el vínculo C.F.P, donde se accede a la página principal (Fig. 1). Los requerimientos mínimos del equipo computacional son: una velocidad de procesador de $200 \mathrm{MHz}$ y una memoria RAM de 64 Mbytes. El procedimiento para calcular flujos de potencia de un sistema particular es el siguiente:

- En el menú Principal, ingresar el nombre del SEP, el número de barras del sistema, de líneas y de transformadores con cambios de derivaciones (TCD).

- $\quad$ Se continúa en el menú Barras, donde se ingresa el Número de la Barra, el tipo de barra (PQ, PV o $\mathrm{SL}$ ), el voltaje en pu, el nombre de la barra, las potencias generada y consumida. Los números complejos se ingresan de la forma indicada en la Tabla 1. 
Tabla 1.- Formato de los números complejos en C.F.P

\begin{tabular}{|c|c|}
\hline $\begin{array}{c}\text { Escritura de un número } \\
\text { complejo }\end{array}$ & $\begin{array}{c}\text { Escritura en } \\
\text { C.F.P. }\end{array}$ \\
\hline $1.0+0 \mathrm{i}$ & 1 \\
\hline $0.001+0.2 \mathrm{i}$ & $0.001+0.2$ \\
\hline $0.001-\mathrm{j} 0.2$ & $0.001-0.2$ \\
\hline
\end{tabular}

- Luego se configura en el menú Líneas, la ubicación, la impedancia serie y admitancia en paralelo (en pu) de cada una de las líneas del sistema en estudio.
- $\quad$ Posteriormente se elige el Método de Cálculo a utilizar, entre ellos: Jacobiano Completo, Desacoplado y Desacoplado Rápido.

Una vez finalizada la simulación, emerge una ventana de resultados, que es posible almacenar en disco duro (Fig. 5). Este software ha sido revisado para comprobar su adecuado funcionamiento en diferentes situaciones [8]. Su comportamiento ha sido ampliamente satisfactorio.

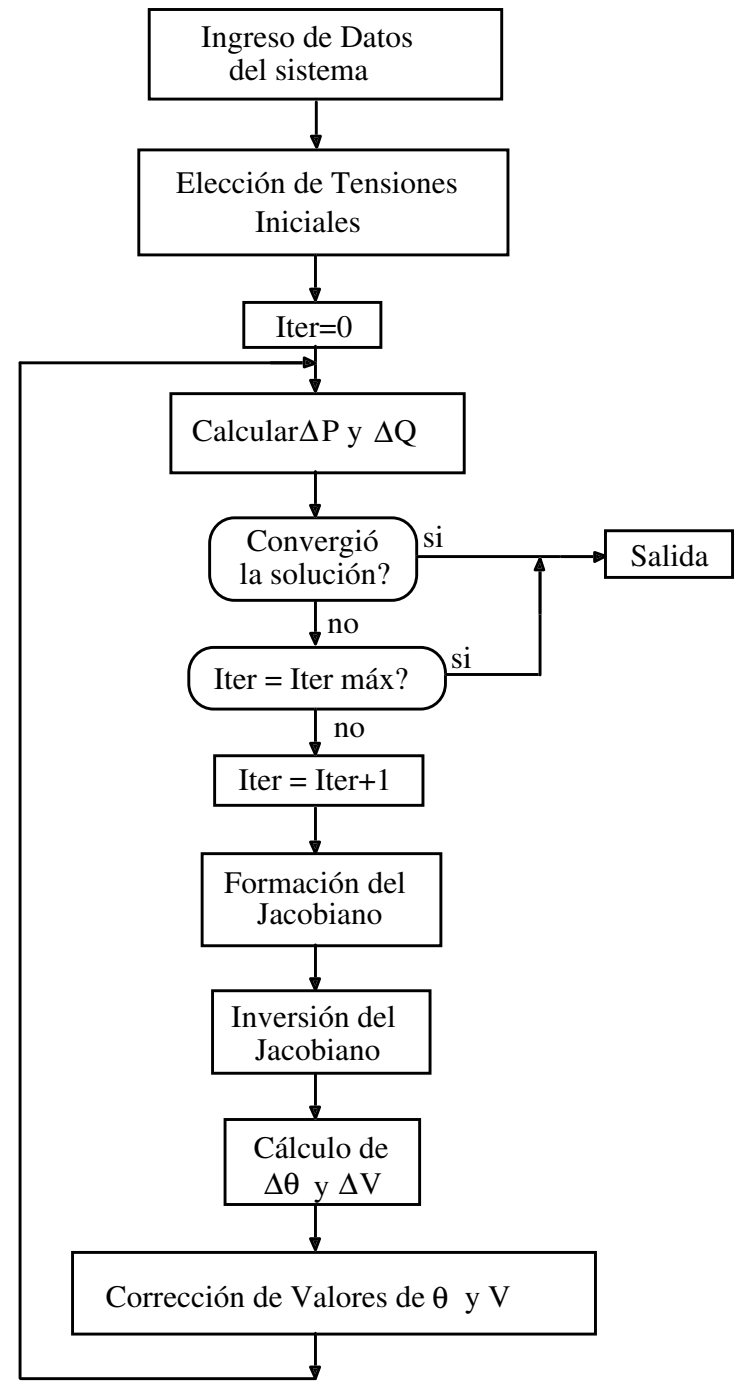

Fig. 4.- Diagrama de Flujo del Algoritmo de Newton-Raphson "Completo" 


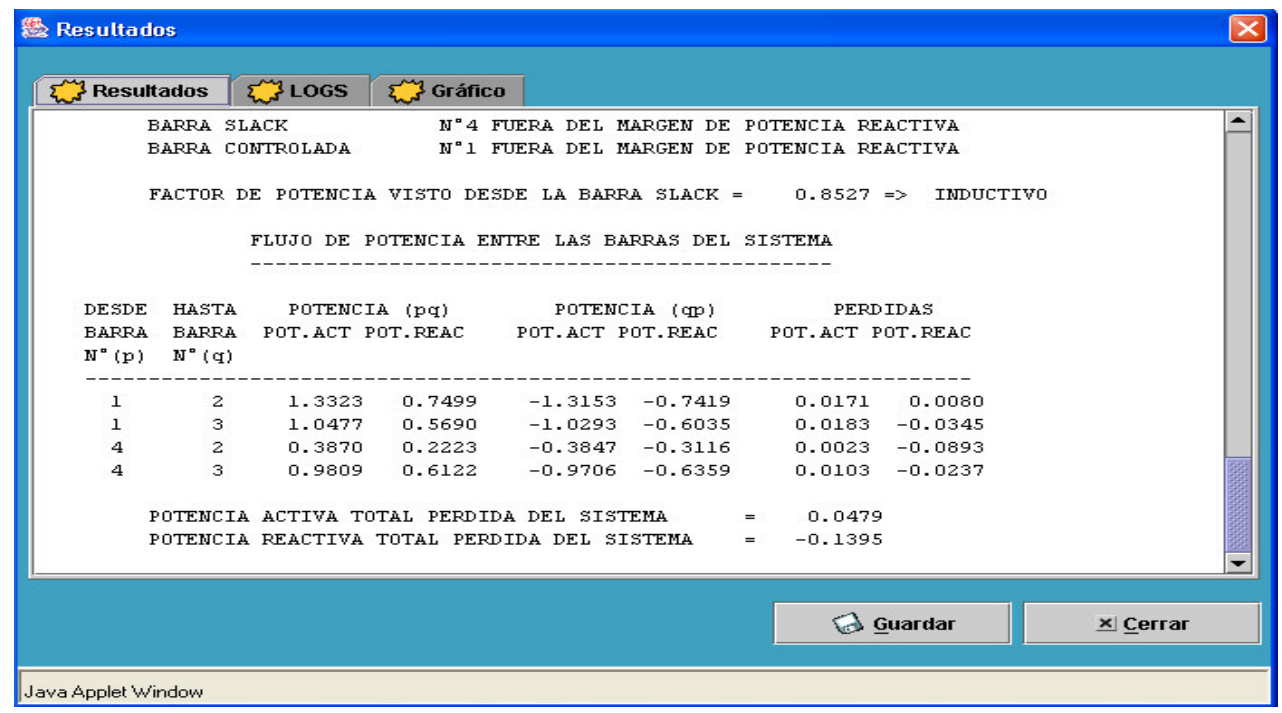

Fig. 5.- Ventana de resultados de simulación de CFP

\section{CONCLUSIONES}

En este trabajo, se presenta el desarrollo de un sitio WEB para el apoyo en la formación de Ingenieros Eléctricos en el área de SEP de la Universidad de La Frontera. Junto a la entrega de conceptos en clases presenciales y a la experimentación en sesiones de laboratorio, se proporciona una herramienta complementaria al proceso formativo, el que considera no sólo los apuntes de clases, guías de ejercicios y de laboratorio, sino que incluye una sección orientada a resolver las preguntas más frecuentes en la asignatura (FAQ) y un software de simulación numérica para el cálculo de flujos de potencia en SEP. El software responde a las exigencias de hoy: confiable, ya que los resultados obtenidos por los métodos numéricos se pueden comparar; interactivo, ya que cuenta con ventanas para el ingreso de datos, versátil ya que es posible revisar datos para agregar o eliminar líneas (o barras), cambiar de posición la barra, modificar las potencias en barras, variar el número de iteraciones y criterio de convergencia; amigable, por contar con ayudas para todas las ventanas y para cada elemento componente; rápido, debido a su velocidad de respuesta y entrega de resultados a través de un listado fácil de analizar; multiplataforma ya que el desarrollo en JAVA permite su ejecución en cualquier sistema operativo; y accesible ya que al estar en un servidor WEB, éste puede ser visitado desde cualquier punto en que se disponga de un acceso a Internet y de un navegador estándar. Este material complementario permite que el estudiante sea el principal actor en su aprendizaje, ya que puede acomodarse a su propio ritmo de estudio. Más aún, la versatilidad de la simulación permite experimentar con situaciones que en clases teóricas o sesiones de laboratorio no es posible realizar por diversas limitaciones. Si bien no ha sido evaluado el impacto real del sitio WEB en los estudiantes de SEP, se contempla como trabajo futuro el estudio de la influencia que éste tiene en el rendimiento de cada uno de los alumnos de la asignatura. Sin embargo, se prevé que los estudiantes se sentirán más motivados, y en consecuencia mejorarán sus calificaciones. Finalmente, se espera que la forma de impartir las clases cambie significativamente de paradigma para facilitar el aprendizaje y mejorar la creatividad de los estudiantes.

\section{REFERENCIAS}

[1] J. Grainger, W. Stevenson Jr.; "Elements of Power System Analysis", Editorial McGraw-Hill, 1997.

[2] G. Stagg, Ahmed H. El-Abiad; "Computer Methods in Power System Analysis", Editorial McGraw-Hill Book Company, 1968.

[3] M. A. Baeza Venegas; "Diseño de un Web turístico para la región de la Araucanía". Trabajo de Titulación, Universidad de la Frontera, 1997. 
[4] M. Villarroel M.; 'Sistemas de Potencias II", Departamento de Ingeniería Eléctrica, Universidad de la Frontera, 2003.

[5] G. Ojeda Rojas, F. Urra Acevedo; 'Cálculo de Flujo de Potencia utilizando métodos de Newton Raphson: Una aplicación de Matlab”. Trabajo de Titulación, Universidad de la Frontera, 1998.

[6] D. Acevedo, A. Sicilia, D. Zurdo; 'Guía rápida de Internet", 1999.

[7] D. Wilmigton; 'Introducción a la programación orientada a objetos", 2000.

[8] J. Chávez Manríquez; 'Diseño de WEB para apoyo docente del curso de Sistemas Eléctricos de Potencia". Trabajo de Titulación, Universidad de la Frontera, 2003. 